Disclaimer: This article has been published immediately upon acceptance (by the Editors of the journal) as a provisional PDF from the revised version submitted by the authors(s). The final PDF version of this article will be available from the journal URL shortly after approval of the proofs by authors(s) and publisher.

\title{
Exact Stability Calculation for Timber Beams and Columns
}

\author{
T. A. C. M. van der Put
}

The Open Construction \& Building Technology Journal, Volume 7, 2013

ISSN: $\quad 1874-8368$

DOI: $\quad 10.2174 / 1874-836820130508001$

Article Type: Review Article

Received: February 19, 2013

Revised: $\quad$ May 03, 2013

Accepted: $\quad$ May 03, 2013

Provisional PDF Publication Date: May 07, 2013

\section{(C) T. A. C. M. van der Put; Licensee Bentham Open.}

This is an open access article licensed under the terms of the Creative Commons Attribution Non-Commercial License (http://creativecommons.org/licenses/ by-nc/3.0/) which permits unrestricted, non-commercial use, distribution and reproduction in any medium, provided the work is properly cited. 


\section{Exact stability calculation for timber beams and columns}

T. A. C. M. van der Put

Faculty of Civil Engineering and Geosciences, Timber Structures and wood technology, TU Delft, P.O. Box 5048, 2600 GA Delft The Netherlands, Tel: +31152851980 e-mail: vanderp@xs4all.nl

Abstract: A derivation is given of simplified, exact stability design rules according to limit analysis, applied to timber beam-columns. These rules are lacking but are necessary to be able to provide real and calculable reliability as is required according to European pacts and laws. Necessary therefore are the obtained exact combined bi-axial bending, compression and shear strength equations with the exact equilibrium equations under biaxial loading. As for other materials the elastic-full plastic limit design approach is applied, which is already known to precisely explain and predict uniaxial bending strength behavior. The strength derivation is based on choosing the location of the neutral line. This provides the stress distribution in the beam cross section in the ultimate state for that case, providing the possibility to calculate the associated ultimate bending moments in both main directions combined with the ultimate normal- and shear forces.

The derived general strength and equilibrium equations are simplified to possible elementary design equations, applicable for building regulation.

keywords: timber beams, limit analysis, stability design, bi-axial bending strength

\section{Introduction}

The exact lower bound equilibrium method of limit analysis is based on finding an allowable equilibrium system, (given in Chapter 3), which nowhere surmounts the failure criterion. This failure criterion, derived in Chapter 2, is an applicable and systematized extension of [1]. Buckling is a three-dimensional problem due to initial eccentricities and because it never is possible to have deformations and loading actions to be precisely zero and thus to get exactly

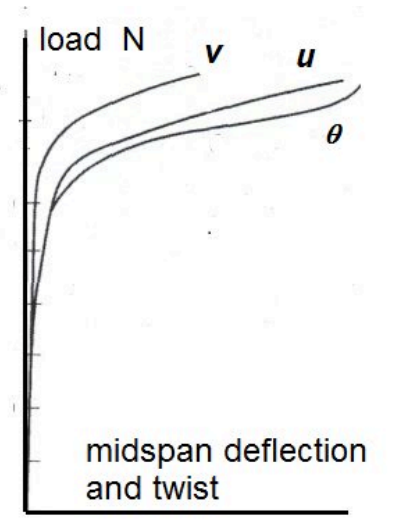
an two-dimensional planar structure and thus to get the possibility of bifurcation. All deformations start at the beginning of loading. Calculations of large deflections of beam-columns by third order theory ([2] p 188) show that there always is a rise of the loading-curve at increasing torque and out of plane deformation, as given by Fig. 1 .

It thus is necessary to have plastic flow, causing stiffness decrease, to bend down the loading curve and the top of the loading curve then represents the ultimate strength which thus is always determining for buckling in practice. Although for a compact strong material, as steel, it should be possible, by machine

Fig.1. Deformation of a beam column according to $3^{\text {rd }}$ order analysis

testing, to go over the top of the loading curve when the test rig - specimen assembly could be made stiff enough, this unloading is not possible by the in practice occurring dead load loading and total failure is shown to occur at the top of the curve (see Fig. 2). Testing at Stevin Laboratory on full structural scale and semi-full scale glulam beams with perfect boundary conditions confirmed this behavior. To investigate buckling behavior at different 


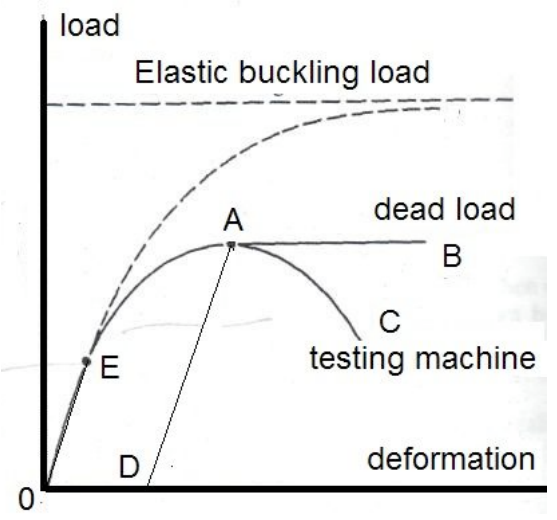

loading conditions, very slender beams, with very low bearing capacities, also were tested. The tests of these slender beams was stopped off at a very small lateral deformation at the point where the lateral rate of deformation started to increase strongly. This point was supposed to be the starting point of bifurcation. However it appeared by the first tests, by the cracking sound and the afterwards measured decrease of the lateral modulus of elasticity, that damage had occurred. This means that the elastic limit (the start of compressional "flow"), thus the start of decrease of the modulus of elasticity, determines buckling behavior and biaxial buckling is plastically and a common ultimate strength problem. The solution thus has to satisfy equilibrium and compatibility conditions, (given

Fig. 2. Scheme of elastic-plastic buckling.

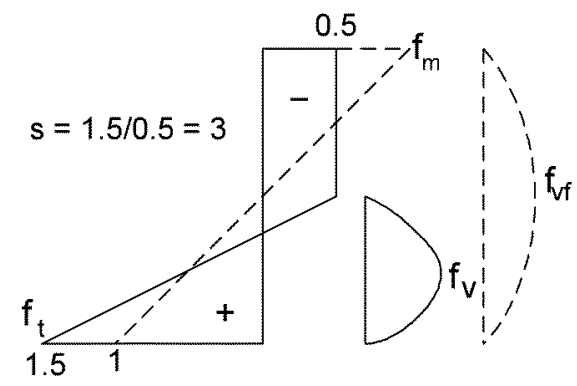

Fig 3. Bending and shear strength

in Chapter 3), and as stress-strain relation, the elastic-full plastic behavior applies, according to limit analysis (thus with an averaging effect of some hardening). For design the ultimate state is important and it is not necessary to follow the loading history dependent loading curve (line EA of Fig. 2) by the descriptive tangent stiffness approach. In practice the linear elastic line DA or 0A of Fig. 2 is followed. For the bending strength of lateral supported beams, the elastic-plastic stress diagram or the ultimate state at point A, is given by the drawn lines in Fig. 3 . At unloading the behavior is linear elastic according to the dashed line in Fig. 3 and the stress difference between the dashed and drawn lines of Fig. 3 gives an internal equilibrium system of the residual stresses after unloading. After reloading, the dashed stress diagram is again superposed, giving the definition of the apparent bending stress $\sigma_{m}$ with the possibility of a linear elastic calculation, according to the dashed line in Fig. 3, up to the ultimate failure state $f_{m}$. It however is necessary to correct the deformation in accordance with the real deformation given by tensile gradient of the drawn lines in Fig. 3. This means that the linear elastic modulus of elasticity has to be reduced by a factor $2 /\left(1+\sigma_{t} / \sigma_{c}\right)$, where $\sigma_{t}$ and $\sigma_{c}$ are the real occurring maximum tensile and compression stresses along the beam. In Fig. 3, of the ultimate state, this factor is: $2 /\left(1+f_{t} / f_{c}\right)=2 /(1+3)=0.5$, showing the real elastic-plastic rotation to be a factor 2 higher with respect to the dashed bending stress diagram. In [3] different possibilities are discussed to account for this compliance increase. In the past this factor 2 was safely accounted for any load distribution in the Building Codes

\section{Exact biaxial bending strength equations}

\subsection{Introduction}

It is a prescribed custom to transform the ultimate strength state of timber beams to linear, quasi isotropic, behavior for the loading case of bending, compression with shear and to apply common beam theory. The consequence of this choice is that fictive bending strength $f_{m}$, 
based on the linearized bending stress in the failure state, given in [4], therefore only applies for rectangular cross-sections (and not for profiles) for the most elementary loading case. For combined loading cases and to explain measurements, the elastic-full plastic diagram (of Fig. 3) has to be used as shown e.g. in [3], where the derivation is given of the uniaxial bending, compression and shear strength of timber beams. For profiles this elastic-plastic approach has to be applied to obtain the necessary profile factors on the fictive linear bending strength $f_{m}$. The elastic-full plastic approach is the basis for limit design and is an exact approach which applies for all materials as is extensively shown for other materials as steel and concrete [2] and provides a critical loading path for real strength prediction. For wood this necessary design method was already generally applied since 1930 (see [5]). Necessary for stability design and for the prescribed calculable reliability, is the hereupon based exact bi-axial bending strength criterion, combined with normal compression and shear loading which is mathematically derived in [6]. The resulting elastic-plastic stress diagram, with a negligible plastic range for tension, as applied in the figures below, represents an admissible equilibrium system, satisfying equilibrium and boundary conditions, violating nowhere the yield criterion, and thus is a lower bound solution of limit analysis. The highest lower bound solution is equal to the real strength and this is reached in this case when the neutral line is a straight line and when unlimited flow in pure compression is possible, thus when the shear stress is carried in the elastic part of the cross section. Thus, as confirmed in [3], the uniaxial ultimate combined bending-compression strength is determined by the ultimate tensile stress $f_{t}$ and by unlimited "flow" in compression at the flow compression stress $f_{c}$. Bending failure thus always is an ultimate tension failure at $f_{t}$. This therefore is the starting point for the derivations in [6] and is an improvement with respect to the old model, applied in [7], which was based on a limited ultimate compression strain and therefore did not explain and fit precisely to the data.

The derivations in [6] of the following equations are based on choosing the location of the neutral line and calculate the associated ultimate bending moments and normal and shear forces. There are three cases to regard for the location of the neutral line. The neutral line may go through two opposite planes of the cross section (Case I) as given in Fig. 4, or the neutral line goes through two adjacent planes, at the tension side (Case II) or at the compression side (Case III) as given by respectively Fig. 5 and 6. Mathematically simpler is not to choose the location of the neutral line but of the parallel border line of the full plastic compression plateau of the cross section as is the basis of the following equations.

\subsection{Bi-axial bending strength cases}

\subsubsection{Dominating bending in the stiff direction (Case $\mathbf{I}: Y \geq b ; Z \leq h$ in Fig. 4)}

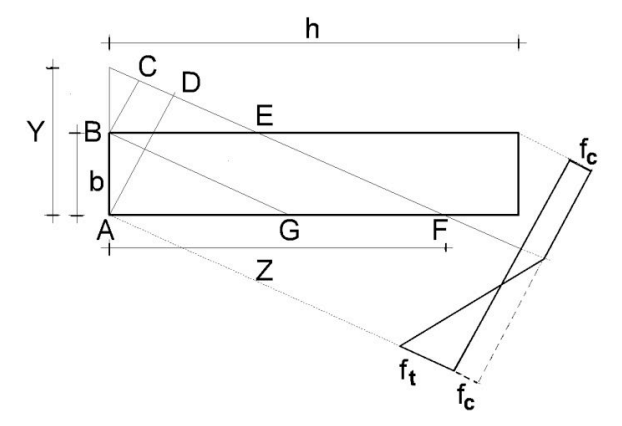

The ultimate state of the determining cross-section of a beam loaded under biaxial bending, given in Fig. 4 for Case I. The line EF in Fig. 4 is the boundary of the full plastic, ultimate compression strength area of the cross-section of a beam. Fig. 4 thus gives an equilibrium state of a beam with dimension $b$ and $h$, loaded in "double" bending. For the analysis, the bending stresses of the ultimate state are regarded to be a superposition of compression force $N_{u}=f_{c} b h$ of the uniform ultimate compression stress $f_{c}$ over the

Fig.4 - Compression with bi-axial bending 
entire cross-section and a tension force by the linear increasing tensile stresses in the plane ABEF with a maximal tensile stress $f_{t}+f_{c}$ in point $\mathrm{A}$ (see the derivation in [6]).

\subsubsection{Normal forces (Case I)}

The ultimate normal force $N_{u}$, with (for convenience) a positive sign for compression, is:

$$
N_{u}=f_{c} b h-T=f_{c} b h-\left(f_{t}+f_{c}\right) \frac{b Z}{2}\left(1-\frac{b}{Y}+\frac{b^{2}}{3 Y^{2}}\right)=f_{c} b h\left[1-(s+1) \frac{Z}{2 h}\left(1-\frac{b}{Y}+\frac{b^{2}}{3 Y^{2}}\right)\right] \ldots
$$

where $s=f_{t} / f_{c}$. Thus, with the maximal possible value of $N_{u, m}=f_{c} b h$ follows for $Z / h$ :

$$
(s+1) \frac{Z}{2 h}\left(1-\frac{b}{Y}+\frac{b^{2}}{3 Y^{2}}\right)=1-\frac{N_{u}}{N_{u, m}} \ldots
$$

For uniaxial bending in the stiff direction, $Y \rightarrow \infty$ (or: $b / Y \rightarrow 0$ ), this equation agrees with Eq.(2) of [3], where it was shown that the theory precisely fits the data of [7]. This case is determining for the possible extreme values of Case I:

$1 \geq N_{u} / N_{u, m} \geq-(s-1) / 2 \ldots$

with a tension limit (negative sign) when $s>1$.

\subsubsection{Bending moments (Case I)}

The bending moment by the tensile stress pyramids in the cross section with respect to the resultant compression force $N_{u, m}=f_{c} b h$, thus with respect to the center of the cross-section of the beam, gives:

$$
M_{y}=\left(f_{t}+f_{c}\right) \frac{b Z h}{4}\left(1-\frac{b}{Y}+\frac{b^{2}}{3 Y^{2}}\right)-\left(f_{t}+f_{c}\right) \frac{b Z^{2}}{12}\left(2-3 \frac{b}{Y}+2 \frac{b^{2}}{Y^{2}}-\frac{b^{3}}{2 Y^{3}}\right) \cdots
$$

and thus Case I applies when: $0 \leq M_{y} \leq f_{c} \frac{(s+1)}{2} \cdot \frac{b h^{2}}{6}$.

Substitution of $Z$ according to Eq.(2) in Eq.(4) gives:

$$
M_{y}=f_{c} \frac{b h^{2}}{6}\left(1-\frac{N_{u}}{N_{u, m}}\right)\left[3-\frac{1-N_{u} / N_{u, m}}{s+1} \cdot \frac{4-6 b / Y+4 b^{2} / Y^{2}-b^{3} / Y^{3}}{\left(1-b / Y+b^{2} /\left(3 Y^{2}\right)\right)^{2}}\right] \ldots
$$

Eq.(5) can be written:

$$
M_{y}=f_{c} \frac{b h^{2}}{6}\left(1-\frac{N_{u}}{N_{u, m}}\right)\left[3-\frac{1-N_{u} / N_{u, m}}{s+1} \cdot \Phi_{1}\left(\frac{b}{Y}\right)\right]
$$

with: $\Phi_{1}(b / Y)=\frac{4-6 b / Y+4 b^{2} / Y^{2}-b^{3} / Y^{3}}{\left(1-b / Y+b^{2} /\left(3 Y^{2}\right)\right)^{2}}$.

In the applying range ( $Z \leq h$ and $b / Y$ between 0 to 1$), \Phi_{1}(b / Y)$ is a bend curve which precisely can be approximated e.g. by parabola: $\Phi_{1}(b / Y)=4+b / Y+4(b / Y)^{2}$, or by a power form: $\Phi_{1}(b / Y)=4+5(b / Y)^{1.8} \ldots$

Thus: $M_{y}=f_{c} \frac{b h^{2}}{6}\left(1-\frac{N_{u}}{N_{u, m}}\right)\left[3-\frac{1-N_{u} / N_{u, m}}{s+1} \cdot\left(4+5\left(\frac{b}{Y}\right)^{1.8}\right)\right] \ldots$

and $b / Y$ is directly known from: $M_{y}$ and $N$, where $M_{y}$ is the component in the stiff direction. 
The condition for application as Case I is:

$0 \leq M_{y} \leq f_{c} \frac{b h^{2}}{6}\left(1-\frac{N_{u}}{N_{u, m}}\right)\left(\frac{3 s-1+4\left(N_{u} / N_{u, m}\right)}{s+1}\right) \leq f_{c} \frac{(s+1)}{2} \cdot \frac{b h^{2}}{6}$

The other component in the weak direction of the biaxial moment is $M_{z}$ :

$M_{z}=\left(f_{c}+f_{t}\right) \frac{b^{3} Z}{12 Y}\left(1-\frac{b}{2 Y}\right) \cdots$

According to the Case I boundary conditions is: $0 \leq M_{z} \leq f_{c} h b^{2}(s+1) / 24 \ldots$

By substitution of $Z$ according to Eq.(2), Eq.(9) becomes:

$M_{z}=f_{c} \frac{h b^{2}}{6} \cdot \frac{b / Y(1-b / 2 Y)}{1-b / Y+b^{2} / 3 Y^{2}} \cdot\left(1-\frac{N_{u}}{N_{u, m}}\right) \cdots$

This equation can be simplified to:

$M_{z}=f_{c} \frac{h b^{2}}{6} \cdot\left(1-\frac{N_{u}}{N_{u, m}}\right) \cdot \Phi_{2}(b / Y)=f_{c} \frac{h b^{2}}{6} \cdot\left(1-\frac{N_{u}}{N_{u, m}}\right) \cdot\left(\frac{b}{Y}+0.5\left(\frac{b}{Y}\right)^{1.8}\right) \ldots$

because $\Phi_{2}(b / Y)=\frac{1-b / 2 Y}{-1+Y / b+b / 3 Y} \approx b / Y+0.644(b / Y)^{2}-0.144(b / Y)^{3}$,

that also can be approximated by the power form: $\Phi_{2}(b / Y)=b / Y+0.5(b / Y)^{1.8} \ldots$

Because for Case I: $Y \geq b ; Z \leq h$, is:

$M_{z} \leq f_{c} \frac{h b^{2}}{4} \cdot\left(1-\frac{N_{u}}{N_{u, m}}\right) \leq f_{c} \frac{h b^{2}}{24}(s+1)$

For $Y \rightarrow \infty, M_{z}=0$ as follows from Eq.(9) or (11) and thus uniaxial bending occurs and Eq. (2) then becomes: $(s+1) Z /(2 h)=1-N_{u} / N_{u, m}$.

When this is substituted in Eq.(4) for $Y \rightarrow \infty$, the uniaxial bending strength becomes:

$M_{y, \infty}=\left(f_{t}+f_{c}\right) \frac{b Z h}{4}-\left(f_{t}+f_{c}\right) \frac{b Z^{2}}{6}=f_{c} \frac{b h^{2}}{6} \cdot \frac{-1+3 s+4 N_{u} / N_{u, m}}{s+1} \cdot\left(1-\frac{N_{u}}{N_{u, m}}\right) \cdots$

as found before in [3], verified by the precise fit to the data of [7].

The fictive linear elastic design bending stress, applied in the Building Codes, thus is:

$\sigma_{m}=\frac{6 M_{y, \infty}}{b h^{2}}=f_{c} \cdot \frac{-1+3 s+4 N_{u} / N_{u, m}}{s+1} \cdot\left(1-\frac{N_{u}}{N_{u, m}}\right) \ldots$

which is equal to the uniaxial bending strength $f_{m}$, given in Fig. 3, when $N=0$, Thus:

$f_{m}=\frac{6 M_{y, \infty}}{b h^{2}}=f_{c} \frac{3 s-1}{s+1} \ldots$

In [3], the value of $s=1.3$ was found for the mean strength, while $s=2$ for the 95th percentile and $s=0.77$ for the 5 th percentile of the uniaxial combined bending compression strength, given in Fig. 3, 4 and 5 of [3], where $m=6 M_{u} / f_{m} b h^{2}$ and $n=N_{u} / f_{c} b h$. These values are based on the data of [8] and apply for the total wood population at standard test conditions. For North European wood $s=1.56$ was found in [9] for timber and $s=2.15$ for veneer wood. In [3], $s=1.67$ is arbitrarily assumed for stability. Higher values apply at high moisture contents. Important is, that these values of $s$ are independent of the load-combination, showing that there is no, (or no strong), volume effect due to tensile stress distribution, but only for volume alone. Tensile failure thus shows some 
plasticity and the volume effect is explained by a decrease of quality with volume increase. This also explains why by not brittle compression failure a volume effect is possible (as reported in literature). Values of $s$ should be controlled by the exact failure criterion [10].

\subsubsection{Shear force (Case I)}

The total ultimate resulting shear force $V_{u}\left(=\sqrt{V_{x}^{2}+V_{y}^{2}}\right)$ in the elastic region of eq.(19) the cross section is:

$V_{u}=\frac{2}{3} f_{v} b Z\left(1-\frac{b}{2 Y}\right) \cdots$

based on the parabolic shear stress distribution in the elastic region. The the possible range of $V_{u}$ is: $0 \leq V_{u} \leq 2 f_{v} b h / 3$ for Case I.

Substitution of $Z$ of Eq.(2) into Eq.(17) gives:

$$
\begin{aligned}
& V_{u}=\frac{2}{3} f_{v} b h\left(1-\frac{b}{2 Y}\right) \cdot\left(\frac{1-N_{u} / N_{u, m}}{1-b / Y+b^{2} /\left(3 Y^{2}\right)}\right) \cdot \frac{2}{s+1} \ldots \\
& V_{0, \infty} \leq \frac{2}{3} f_{v} b h \frac{2}{s+1}\left(1-N_{u} / N_{u, m}\right) \leq \frac{2}{3} f_{v} b h
\end{aligned}
$$

or with uniaxial $V_{0, \infty}$ according to Eq.(20):

$$
\frac{V_{u}}{V_{0, \infty}}=\left(1-\frac{b}{2 Y}\right)\left(\frac{1-N_{u} / N_{u, m}}{1-b / Y+b^{2} /\left(3 Y^{2}\right)}\right) \cdots
$$

The shear strength $V_{0, \infty}$ is determined at ultimate uniaxial bending, for $N=0$ and $Y \rightarrow \infty$ and is given for design as a fictive linear elastic parabolic stress distribution over the total depth $h$, with topvalue $f_{v, f}$, according to Fig. 3. Thus

$V_{0, \infty}=\frac{2}{3} f_{v} b h \frac{2}{s+1}=\frac{2}{3} f_{v, f} b h \ldots$

For $Y \rightarrow \infty$, Eq.(19) represents the uniaxial loading case (see [3]) giving:

$\frac{V_{u, \infty}}{V_{0, \infty}}=1-\frac{N_{u}}{N_{u, m}} \ldots$

According to Eq.(19) is for biaxial loading :

$\frac{V_{u}}{V_{0, \infty}}=\left(1-\frac{N_{u}}{N_{u, m}}\right) \Phi_{3}(b / Y)$ with: $\Phi_{3}(b / Y)=\frac{1-b / 2 Y}{1-b / Y+b^{2} /\left(3 Y^{2}\right)}=\frac{\Phi_{2}(b / Y)}{b / Y}$.

Thus:

$$
\frac{V_{u}}{V_{0, \infty}}=\left(1-\frac{N_{u}}{N_{u, m}}\right) \cdot\left(1+0.5\left(\frac{b}{Y}\right)^{0.8}\right) \ldots
$$

\subsubsection{Dominating bending in the weak direction (Subcase I): $Z \geq h$ and $Y \leq b$ )}

For $Z \geq h$ and $Y \leq b$, the same equations of Section 2.2.1 apply with interchange of $z$ and $y$; $Z$ and $Y, b$ and $h$.

Again one component of the biaxial moment shows the linear relation with $\left(1-N / N_{u, m}\right)$.

2.2.3. Dominating high compression and high biaxial bending ( Case II: $Y \leq b ; Z \leq h$ in Fig. 5) 


\subsubsection{Normal forces (Case II)}

$N_{u}=f_{c} b h-\frac{1}{2} Z Y \cdot \frac{1}{3}\left(f_{t}+f_{c}\right)=f_{c} b h\left(1-\frac{s+1}{6} \cdot \frac{Y Z}{b h}\right) \quad$ or: $\quad \frac{s+1}{6} \cdot \frac{Y Z}{b h}=1-\frac{N_{u}}{N_{u, m}} \ldots$

According to the boundary conditions is $((5-s) / 6) \leq N_{u} / N_{u, m} \leq 1 \ldots$

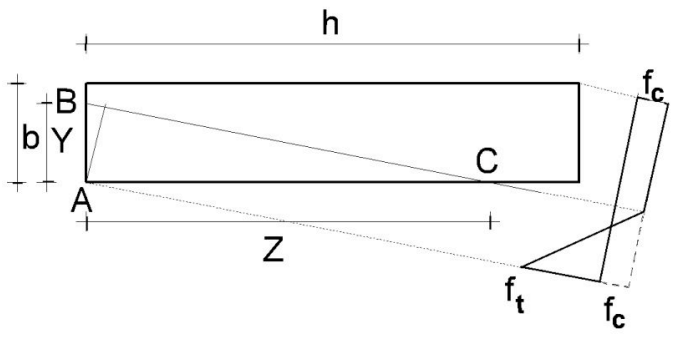

Fig. 5. Dominating compression with bi-axial bending for $Z \leq h$ and $Y \leq b$

\subsubsection{Shear force (Case II)}

For the ultimate total shear force applies:

$V_{u}=\frac{2}{3} f_{v} \frac{Y Z}{2}=f_{v} \frac{Y Z}{3}=f_{v} b h \frac{2}{s+1}\left(1-\frac{N_{u}}{N_{u, m}}\right) \cdots$

Thus, for determining shear strength, is, due to the boundary conditions $Z \leq h$ and $Y \leq b$ :.

$V_{u} \leq f_{v} b h / 3 \ldots$

because $N_{u} / N_{u, m}$ cannot be lower than: $N_{u} / N_{u, m} \geq((5-s) / 6)$, according to Eq. (24).

\subsubsection{Bending moments (Case II)}

As before, the resultant force of the tensile stress pyramid times the distance to the resultant compression force in the center of the cross section determines the bending moment.

For bending applies, using Eq.(23):

$$
\begin{aligned}
& M_{y}=\left(f_{c}+f_{t}\right) \frac{Y Z}{6}\left(\frac{h}{2}-\frac{Z}{4}\right)=f_{c} b h\left(\frac{h}{2}-\frac{Z}{4}\right)\left(1-\frac{N_{u}}{N_{u, m}}\right) \leq f_{c} b h \frac{h}{4}\left(1-\frac{N_{u}}{N_{u, m}}\right) \leq f_{c}(s+1) \frac{b h^{2}}{24} . . \\
& M_{z}=\left(f_{c}+f_{t}\right) \frac{Y Z}{6}\left(\frac{b}{2}-\frac{Y}{4}\right)=f_{c} b h\left(\frac{b}{2}-\frac{Y}{4}\right)\left(1-\frac{N_{u}}{N_{u, m}}\right) \leq f_{c} b h \frac{b}{4}\left(1-\frac{N_{u}}{N_{u, m}}\right) \leq f_{c}(s+1) \frac{h b^{2}}{24}
\end{aligned}
$$

Knowing $M_{y}, N$ and $M_{z}, \mathrm{Y}$ and $\mathrm{Z}$ are known and the found product $Y Z$ should be smaller for determining bending failure than the value of $Y Z$ for shear failure according to Eq.(25), thus $(Y Z)_{\text {bending }} \leq\left(3 V_{u} / f_{v}\right) \ldots$

2.2.4. Dominating high biaxial bending with moderate normal force (Case III: $Y \geq b$; $Z \geq h$ in Fig. 6)

For dominating tension, the condition $Y \geq b$ and $Z \geq h$ may apply according to Fig. 6 . 


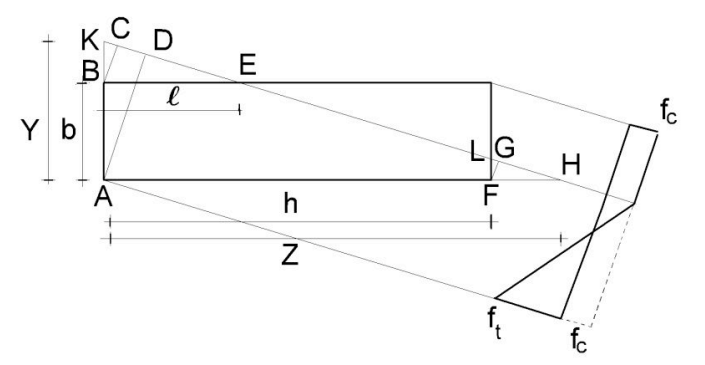

Fig. 6. Dominating tension with bi-axial bending for $Z \geq h$ and $Y \geq b$

\subsubsection{Normal forces (Case III)}

From the equilibrium equations follows that the ultimate normal compression force is:

$N_{u}=f_{c} b h-T_{u}=f_{c} b h\left(1-\frac{s+1}{6} \frac{Y Z}{b h}\left(1-\left(1-\frac{b}{Y}\right)^{3}-\left(1-\frac{h}{Z}\right)^{3}\right)\right) \ldots$

To replace $Y Z$ in other equations, this can be written with $N_{u, m}=f_{c} b h$ :

$\frac{s+1}{6} \frac{Y Z}{b h}\left(1-\left(1-\frac{b}{Y}\right)^{3}-\left(1-\frac{h}{Z}\right)^{3}\right)=1-\frac{N_{u}}{N_{u, m}} \ldots$

For applicability in this range: $\frac{5-s}{6} \geq \frac{N_{u}}{N_{u, m}}=1-\frac{s+1}{6} \frac{Y Z}{b h}\left(1-\left(1-\frac{b}{Y}\right)^{3}-\left(1-\frac{h}{Z}\right)^{3}\right) \geq-\frac{s-1}{2}$

In the limit case of Eq.(31) is for: $h=Z, Y \rightarrow \infty$ (or $b / Y \rightarrow 0)$ :

$\frac{s+1}{6} \frac{Y}{b}\left(1-\left(1-\frac{b}{Y}\right)^{3}\right)=\frac{s+1}{6} \frac{Y}{b}\left(1-\left(1-3 \frac{b}{Y}+3\left(\frac{b}{Y}\right)^{2}-\left(\frac{b}{Y}\right)^{3}\right)\right) \approx \frac{s+1}{2}=1-\frac{N_{u}}{N_{u, m}} \ldots$

The same applies for $b=Y, Z \rightarrow \infty$.

\subsubsection{Shear force (Case III)}

The ultimate shear force is:

$V_{u}=\frac{2}{3} f_{v} b h\left(1-\frac{\left(b-\frac{Z-h}{Z} Y\right)\left(h-\frac{Y-b}{Y} Z\right)}{2 b h}\right)=\frac{2}{3} f_{v} \frac{b h}{2}\left(\frac{2 Y}{b}+\frac{2 Z}{h}-\frac{h Y}{b Z}-\frac{b Z}{h Y}-\frac{Z Y}{b h}\right) \cdots$

In the limit case is for: $(h=Z, Y \rightarrow \infty)$ or for $(b=Y, Z \rightarrow \infty): V_{u}=2 f_{v} b h / 3$, and $V_{u}=f_{v} b h / 3$ for $(h=Z, b=Y)$. Thus when shear is determining is: $2 f_{v} b h / 3 \geq V_{u} \geq f_{v} b h / 3$

\subsubsection{Bending moments (Case III)}

The ultimate bending moment is:

$$
M_{y}=f_{c} \frac{(s+1)}{2} \cdot \frac{Y Z h}{6} \cdot\left(1-\left(1-\frac{b}{Y}\right)^{3}-\left(1-\frac{h}{Z}\right)^{3}-\frac{Z}{2 h}+\frac{Z}{2 h}\left(1-\frac{b}{Y}\right)^{4}+\left(1-\frac{h}{Z}\right)^{3}\left(\frac{3}{2}+\frac{Z}{2 h}\right)\right) \ldots
$$

For the limit case in accordance with Eq.(36): $h=Z, Y \rightarrow \infty$ applies for $M_{y}$, giving: 
$M_{y}=f_{c} \frac{s+1}{2} \frac{b h^{2}}{6}$, or half this value when $b=Y, h=Z \ldots$

The limit case: $b=Y, Z \rightarrow \infty$ applies to $M_{Z}$ leading to

$M_{z}=f_{c} \frac{s+1}{2} \cdot \frac{h b^{2}}{6}$, or: when $b=Y, h=Z: M_{z}=f_{c} \frac{s+1}{2} \cdot \frac{h b^{2}}{12} \ldots$

In general is $M_{z}$ :

$M_{z}=f_{c} \frac{s+1}{12} Y Z b\left(\left(1-\left(1-\frac{b}{Y}\right)^{3}-\left(1-\frac{h}{Z}\right)^{3}\right)-\frac{Y}{2 b}+\left(1-\frac{b}{Y}\right)^{3}\left(\frac{3}{2}+\frac{Y}{2 b}\right)+\left(1-\frac{h}{Z}\right)^{4} \frac{Y}{2 b}\right) \ldots$

\subsubsection{High tensional loading (Subcase III)}

The equations o Case III can not strongly be simplified and should be tabulated for different values of $h / Z$ and $b / Y$ or solved by a numerical method for a given loading. Because for high tension and for lower qualities and large structural sizes the (long term) tensile strength will be lower than the compression strength and the behavior is linear elastic, based on the ultimate tensile stress leading to:

$f_{t}=\frac{6 M_{y}}{b h^{2}}+\frac{6 M_{z}}{h b^{2}}+\frac{N_{u}}{b h} \quad$ or: $1=\frac{M_{y}}{M_{y, u}}+\frac{M_{z}}{M_{z, u}}+\frac{N_{u}}{N_{t, u}} \ldots$

In this equation tension has the positive sign.

\subsection{Conditions for design equations}

The given equations for biaxial bending are easy programmable for numerical solutions.

However it always is necessary to provide simple exact Code rules.

The boundary conditions of application of the equations are determined by the uniaxial bending cases and therefore for the following conditions.

\subsubsection{Loading conditions}

Loading conditions for application of Case I equations:

for: $Y \geq b ; Z \leq h$ in Fig. 4:

$$
\begin{aligned}
& 1 \geq N_{u} / N_{u, m} \geq-(s-1) / 2 . \\
& 0 \leq M_{y} \leq f_{m} \frac{b h^{2}}{6}\left(1-\frac{N_{u}}{N_{u, m}}\right)\left(\frac{3 s-1+4\left(N_{u} / N_{u, m}\right)}{3 s-1}\right) \leq f_{c} b h^{2} \frac{(s+1)}{12} \\
& 0 \leq M_{z} \leq f_{c} h b^{2}\left(1-N_{u} / N_{u, m}\right) / 4 \leq f_{c} b h^{2}(s+1) / 24 \\
& V_{u} \leq f_{v} b h \frac{2}{s+1}\left(1-\frac{N_{u}}{N_{u, m}}\right)=\frac{2}{3} f_{v, f} b h\left(1-\frac{N_{u}}{N_{u, m}}\right)=V_{0, \infty}\left(1-N_{u} / N_{u, m}\right) \leq \frac{2}{3} f_{v} b h
\end{aligned}
$$

In all equations is $f_{c}=f_{m}(s+1) /(3 s-1)$ and $f_{v}=f_{v, f}(s+1) / 2$, where $f_{m}$ and $f_{v, f}$ are the linearized values of the design regulations as the Eurocode.

For dominating bending in the weak direction, (Subcase I: $Z \geq h$ and $Y \leq b$ ), $b$ and $h$ should be exchanged in the Case I equations above. 
The loading conditions for application of Case II equations are:

for: $Y \leq b ; Z \leq h$ in Fig. 5

$$
\begin{aligned}
& ((5-s) / 6) \leq N_{u} / N_{u, m} \leq 1 \\
& V_{u} \leq f_{v} b h / 3, \\
& M_{y} \leq f_{c} b h^{2}\left(1-N_{u} / N_{u, m}\right) / 4 \leq f_{c} b h^{2}(1+s) / 24, \\
& M_{z} \leq f_{c} h b^{2}\left(1-N_{u} / N_{u, m}\right) / 4 \leq f_{c} h b^{2}(1+s) / 24 .
\end{aligned}
$$

The loading conditions for application of Case III equations are:

for: $Y \geq b ; Z \geq h$ in Fig. 6

$$
\begin{aligned}
& -(s-1) / 2 \leq N_{u} / N_{u, m} \leq((5-s) / 6) \\
& f_{v} b h / 3 \leq V_{u} \leq 2 f_{v} b h / 3, \\
& f_{c} b h^{2}(s+1) / 24 \leq M_{y} \leq f_{c} b h^{2}(s+1) / 12, \\
& f_{c} h b^{2}(s+1) / 24 \leq M_{z} \leq f_{c} h b^{2}(s+1) / 12
\end{aligned}
$$

The condition for application of Subcase III for high a tension load $N_{u}$ is the ultimate state:

$$
\frac{6 M_{y}}{b h^{2}}+\frac{6 M_{z}}{h b^{2}}+\frac{N_{u}}{b h} \leq f_{t} \quad \text { or: } \frac{M_{y}}{M_{y, u}}+\frac{M_{z}}{M_{z, u}}+\frac{N_{u}}{N_{t, u}} \leq 1 .
$$

Tension has the positive sign in this equation. ))

\subsubsection{Linearized $M-N$ design equations}

All equations show a linear relation with the normal force $N$, except for the case of dominating bending in the main direction, Eq.(6), combined with low shear loading. For design, Eq.(6) therefore should be linearized and can be written, using Eq.(16):

$$
\frac{M_{y}}{f_{m} b h^{2} / 6}=\left(1-\frac{N}{N_{u, m}}\right)\left[\frac{(3 s+3)-\left(1-N / N_{u, m}\right) \Phi_{1}(b / Y)}{3 s-1}\right] \text {, }
$$

where $f_{m}$ is the fictive bending strength of the Eurocode. This equation will be written:

$$
m_{y}=(1-n)\left(\frac{(3 s+3)-(1-n) \Phi_{1}}{3 s-1}\right)=\frac{3 s+3}{3 s-1}(1-n)-(1-n)^{2} \frac{\Phi_{1}}{3 s-1} \ldots
$$

with $\Phi_{1}(b / Y)=4+5(b / Y)^{1.8}$. Similarly Eq.(12) can be written:

$m_{z}=(1-n) \cdot \frac{s+1}{3 s-1} \Phi_{2} \ldots$

with $\Phi_{2}(b / Y)=b / Y+0.5(b / Y)^{1.8}$. This linear relation between $m$ and $n$, also applies for the shear strength Eq.(21).

The bending strength is measured on slender beams with a span to depth ratio over 7 to 8 in a 3-point bending test. Then is: $V_{u} a=M_{u}$, where $a=L / 2$ is the distance of the load in the middle of the beam to the support. Thus $a / h=M_{u} / V_{u} h$ is the shear-slenderness with critical value: $a_{c} / h=\left(f_{m} b h^{2} / 6\right) /\left((2 / 3) f_{v, f} b h^{2}\right)=f_{m} / 4 f_{v, f}$.

This critical value were below the bending stress decreases due to the shear loading and of where of maximal bending and maximal shear failure occur at the same time is $a_{c} / h \approx 3$, providing the test-beam dimensions of the shear strength test for mean quality European softwoods. Eq.(21) thus may be written: 


$$
\begin{aligned}
& \frac{V_{u, \infty}}{V_{0, \infty}}=\frac{M_{y} a_{c}}{M_{y, \infty} a}=\frac{3 h M_{y}}{M_{y, \infty} a}=1-\frac{N}{N_{u, m}} \text { or: } \\
& n=1-(3 h / a) m_{y} \ldots
\end{aligned}
$$

For $a=3 h$, the boundary is reached where below the maximal possible bending moment will be reduced by the maximal possible shear force and Eq.(41) then becomes:

$n=1-m_{y} \ldots$

This linear relation was the basis of the Dutch Code [8] and for a new proposal for the Eurocode, and should apply for all Codes as long as the shear calculation according to Eq.(41) is absent. Eq.(41) shows the parabolic Eurocode line to be a factor 2 too unsafe when $a=3 h$ (see Fig. 7 and [3]).

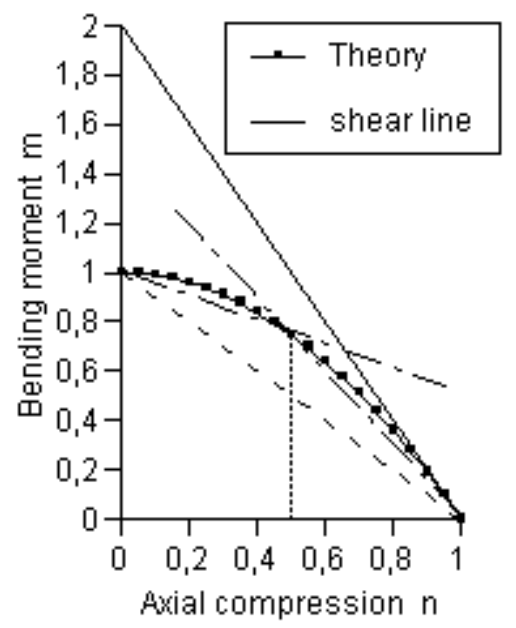

The curved line Eq.(39) the best also can be approximated by 2 straight lines through the end points $(m=1 ; n=0)$ and $(m=$ $0 ; n=1$ ), and through the point on the curve for $\mathrm{n}=0.5$ (see [3]) where according to Eq.(39):

$m_{y}=0.5 \frac{3 s+3-\Phi_{1} / 2}{3 s-1}$,

for $n=0.5$, giving:

$m_{y}=1-\frac{3 s-5+\Phi_{1} / 2}{3 s-1} n \quad$ when $n \leq 0.5$,

and:

$$
n=1-\frac{3 s-1}{3 s+3-\Phi_{1} / 2} m_{y} \quad \text { when } n \geq 0.5 \text {. }
$$

Fig. 7. Interaction curve cut off (by the dashed bending- compression strength shear line or no cut off by the drawn ultimate shear line)

The same equations apply for dominating bending in the weak direction after interchange of $b$ with $h$ (and $Y$ with $Z$ and $y$ with $z$ ).

\subsection{Design procedure and equations}

According to the derivation of the ultimate strength equations in Chapter 2, three cases are possible, depending on the location of the neutral line in the critical cross section. See the notations, for the meaning of the symbols and variables.

\subsubsection{Loading conditions}

The loading conditions for application of Case I equations when: $Y \geq b ; Z \leq h$ (Fig. 4) are: $1 \geq n \geq-(s-1) / 2$.

The negative sign of $n$ stands for tensile stress.

$$
\begin{aligned}
& 0 \leq m_{y} \leq(1-n)\left(\frac{3 s-1+4 n}{3 s-1}\right) \leq \frac{(s+1)^{2}}{2(3 s-1)} \\
& 0 \leq m_{z} \leq(1-n) \frac{(s+1)}{(3 s-1)} \cdot \frac{3}{2} \leq \frac{(s+1)^{2}}{4(3 s-1)} \\
& V_{u} \leq \frac{2}{3} f_{v} b h \frac{2}{s+1}(1-n)=\frac{2}{3} f_{v, f} b h(1-n)=V_{0, \infty}(1-n)
\end{aligned}
$$


In all equations is $f_{c}=f_{m}(s+1) /(3 s-1)$ and $f_{v}=f_{v, f}(s+1) / 2$, where $f_{m}$ and $f_{v, f}$ are the linearized values of the design regulations as the Eurocode.

For dominating bending in the weak direction, (Subcase I: $Z \geq h$ and $Y \leq b$ ), $b$ and $h$ should be exchanged in the Case I equations above.

The loading conditions for application of Case II equations are for: $Y \leq b ; Z \leq h$ (Fig. 5):

$((5-s) / 6) \leq n \leq 1$

$V_{u} \leq f_{v} b h / 3=f_{v f}(s+1) b h / 6=V_{0, \infty}(s+1) / 4$

$m_{y} \leq \frac{(s+1)}{(3 s-1)} \cdot \frac{3(1-n)}{2} \leq \frac{(s+1)^{2}}{4(3 s-1)}$,

$m_{z} \leq \frac{(s+1)}{(3 s-1)} \cdot \frac{3(1-n)}{2} \leq \frac{(s+1)^{2}}{4(3 s-1)}$,

The loading conditions for application of Case III equations are ( $Y \geq b ; Z \geq h$, Fig. 6):

$-(s-1) / 2 \leq n \leq((5-s) / 6)$

The negative sign of $n$ stands for tensile stress

$V_{0, \infty}(s+1) / 4 \leq V_{u} \leq V_{0, \infty}(s+1) / 2$,

$\frac{(s+1)^{2}}{4(3 s-1)} \leq m_{y} \leq \frac{(s+1)^{2}}{2(3 s-1)}$,

$\frac{(s+1)^{2}}{4(3 s-1)} \leq m_{z} \leq \frac{(s+1)^{2}}{2(3 s-1)}$

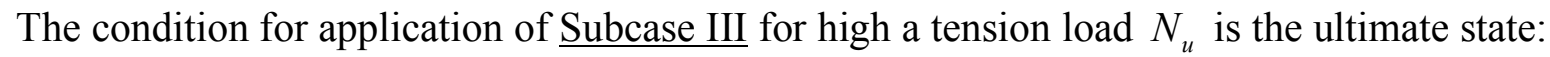
$\frac{6 M_{y}}{b h^{2}}+\frac{6 M_{z}}{h b^{2}}+\frac{N_{u}}{b h} \leq f_{t} \quad$ or: $m_{y}+m_{z}+n \leq 1$.

Where the bending strength $f_{m}$ is equal to the tensile strength $f_{t}$ Tension has the positive sign in this last equation.

\subsubsection{Design equations}

For Case I: ( $Y \geq b ; Z \leq h$, in Fig. 4), of dominating bending in the stiff direction, applies:

$m_{y}=1-\frac{3 s-5+\Phi_{1} / 2}{3 s-1} n \quad$ when $n \leq 0.5 \ldots$

$n=1-\frac{3 s-1}{3 s+3-\Phi_{1} / 2} m_{y} \quad$ when $n \geq 0.5 \ldots$

with $\Phi_{1}=4+5(b / Y)^{1.8}$.

$m_{z}=(1-n) \cdot \frac{s+1}{3 s-1} \Phi_{2} \ldots$

with $\Phi_{2}=b / Y+0.5(b / Y)^{1.8}$,

based on the critical loading according to Chapter 3. From these equations $b / Y$ can be found and dimensions $b$ and $h$ can be adapted. The value of $Z / h$ then follows from: 


$$
(s+1) \frac{Z}{2 h}\left(1-\frac{b}{Y}+\frac{b^{2}}{3 Y^{2}}\right)=1-n \ldots
$$

If the ultimate shear loading $V_{u}=V_{u y}{ }^{2}+V_{u z}^{2}$ is determining, $b / Y$ follows from:

$$
V_{u}=\frac{2}{3} f_{v} b Z\left(1-\frac{b}{2 Y}\right)=(1-n) \cdot\left(1+0.5\left(\frac{b}{Y}\right)^{0.8}\right) V_{0, \infty} \ldots
$$

with: $V_{0, \infty}=\frac{2}{3} f_{v} b h \frac{2}{s+1}=\frac{2}{3} f_{v, f} b h$,

as ultimate uniaxial shear force with $f_{v, f}$ as linearized ultimate shear stress of the Codes.

For uniaxial loading is $b / Y=0$ and eq.(17) turns to:

$V_{u, \infty} / V_{0, \infty}=1-n \ldots$

The uniaxial shear strength also is determining when:

$n=1-(3 h / a) m_{y} \ldots$

where for the test beam: $a / h=M_{u} / V_{u} h=\left(6 f_{m} / b h^{2}\right) /\left(2 f_{v f} b h^{2} / 3\right)=f_{m} /\left(4 f_{v f}\right)$

For Subcase I, $(Z \geq h$ and $Y \leq b)$, for dominating bending in the weak direction, the same equations as Case I apply, with interchange of $z$ and $y ; Z$ and $Y, b$ and $h$.

For Case II: ( $Y \leq b ; Z \leq h$ in Fig. 5), of high biaxial bending and compression, applies:

$$
\begin{aligned}
& m_{y}=\frac{s+1}{3 s-1}\left(3-\frac{3 Z}{2 h}\right)(1-n) \ldots \\
& m_{z}=\frac{s+1}{3 s-1}\left(3-\frac{3 Y}{2 b}\right)(1-n) \ldots
\end{aligned}
$$

From these two equations, $h / Z$ and $b / Y$ are known and can be controlled by:

$\frac{s+1}{6} \cdot \frac{Y Z}{b h}=1-n \ldots$

When the shear strength is determining $Y Z$ follows from:

$V_{u}=f_{v} \frac{Y Z}{3}=f_{v} b h \frac{2}{s+1}(1-n)=f_{v, f} b h(1-n)=\frac{3}{2} V_{0, \infty}(1-n) \ldots$

where $n \geq((5-s) / 6)$

For Case III ( $Y \geq b ; Z \geq h$ in Fig. 6) of biaxial bending with moderate normal force, is:

$$
\begin{aligned}
& \frac{s+1}{6} \frac{Y Z}{b h}\left(1-\left(1-\frac{b}{Y}\right)^{3}-\left(1-\frac{h}{Z}\right)^{3}\right)=1-n \ldots \\
& V_{u}=\frac{2}{3} f_{v} \frac{b h}{2}\left(\frac{2 Y}{b}+\frac{2 Z}{h}-\frac{h Y}{b Z}-\frac{b Z}{h Y}-\frac{Z Y}{b h}\right)=V_{0, \infty} \frac{s+1}{4}\left(\frac{2 Y}{b}+\frac{2 Z}{h}-\frac{h Y}{b Z}-\frac{b Z}{h Y}-\frac{Z Y}{b h}\right) \ldots \\
& m_{y}=\frac{3(s+1)}{3 s-1} \cdot(1-n)\left(1+\frac{-\frac{Z}{2 h}+\frac{Z}{2 h}\left(1-\frac{b}{Y}\right)^{4}+\left(1-\frac{h}{Z}\right)^{3}\left(\frac{3}{2}+\frac{Z}{2 h}\right)}{1-\left(1-\frac{b}{Y}\right)^{3}-\left(1-\frac{h}{Z}\right)^{3}}\right) \ldots
\end{aligned}
$$




$$
m_{z}=\frac{3(s+1)}{3 s-1} \cdot(1-n)\left(1+\frac{-\frac{Y}{2 b}+\frac{Y}{2 b}\left(1-\frac{h}{Z}\right)^{4}+\left(1-\frac{b}{Y}\right)^{3}\left(\frac{3}{2}+\frac{Y}{2 b}\right)}{1-\left(1-\frac{b}{Y}\right)^{3}-\left(1-\frac{h}{Z}\right)^{3}}\right) \ldots
$$

Simplification by first eliminating $h / Z$ is not a right solution because ii leads to extended complicated equations. Thus trial and error solutions are necessary to find $h / Z$ and $b / Y$. Optimal dimensions for $\mathrm{b}$ and $\mathrm{h}$ can be chosen by equating: $m_{y}=m_{z}$ or: $b / Y=h / Z$, which then are directly solvable. Else, if $b$ and $h$ are not free to choose, it provides the best start as initial value of $b / Y=h / Z$ of the iteration process. Multiplying successively both variables with the same small factor give a parallel shift of the neutral line and multiplying one variable with the reverse of factor of the other variable will cause a rotation of the neutral line.

For Subcase III for high biaxial bending with a high tensile load $N_{u}$ applies:

$\frac{6 M_{y}}{b h^{2}}+\frac{6 M_{z}}{h b^{2}}+\frac{N_{u}}{b h} \leq f_{t} \quad$ or: $\frac{M_{y}}{M_{y, u}}+\frac{M_{z}}{M_{z, u}}+\frac{N_{u}}{N_{t, u}} \leq 1$. Thus: $m_{y}+m_{z}+n_{t} \leq 1 \ldots$

where tension has the positive sign in this equation. The same equation applies for first flow when $f_{t}$ is replaced by $f_{c}$, where compression then has the positive sign.

Because by lack of knowledge the coupling of normal force with the ultimate shear force (as given by eqs. $(21,25,41))$ is not accepted in international Codes. Thus for sufficient reliability, the lower bound value of these equation has to be accounted leading to $m_{y}+n=1$ and $m_{x}+n=1$ for uniaxial bending cases. For biaxial loading, when the maximal bending tensile stress occurs at one point this condition becomes: $m_{y}+m_{x}+n=1$.

This eq.(38) was therefore applied for the Dutch design Code TGB, derived and discussed in [11] and [3], using the exact equilibrium equations of Chapter 3, and was applied in many other building regulations and by the old still acceptable version of the Eurocode. This equation and design method thus is approved during many decades (since 1972) as the Dutch Building regulation, TGB.

The resultant bending curvature radius $R$ in the ultimate state region follows from:

$$
\frac{d \varphi}{d x}=\frac{1}{R}=\frac{\varepsilon_{t}+\varepsilon_{c}}{\ell_{2}}=\frac{f_{t}+f_{c}}{E \ell_{2}}
$$

where $\ell_{2}$ is the distance AD in Fig.4, as extension of th uni-axial value discussed in [2], $\ell_{2}=\frac{Y Z}{\sqrt{Y^{2}+Z^{2}}}$

\section{Exact equilibrium equations of beams under biaxial loading}

\subsection{Introduction}

The last proposed design rules of the Eurocode for lateral buckling are not general and consistent and not based on exact theory and thus, as shown, are inherently totally not able to provide real and sufficient reliability in all circumstances. A general, exact, approach is therefore discussed here of the buckling and twist-bend buckling problem of symmetrical profiles loaded in bending in the two main directions and at the same time loaded in torsion and compression. Accounted is for double eccentrically lateral loading, for instance by purlin hangers, in combination with bending in the horizontal direction (wind loading etc.), and for 
the influence of the initial eccentricities, the warping rigidity and the failure criterion. The failure behavior, treated in Chapter 2, is converted to apparent linear behavior until fracture, because empirical strength is expressed in this way. Local buckling of thin webs and flanges is assumed to be prevented by stiffeners. The stability calculation for this last case is provided separately in the Eurocode [4].

The here given derivation is based on an extension of the general differential equations for pure bending with compression of Chen and Atsuta [2], by adding double eccentrically applied lateral loading in 1981, which is applied e.g. in [11]. This is in accordance with the same extension of [2] since 2009. These equations account for the influence of warping and the Wagner effect. (The Wagner effect is the torsional moment appearing by the components of the normal stresses in a warped cross section).The solution of the equilibrium differential equations is based on the virtual work equation, what also is the basis of the rigorous upperand lower bound theorems of limit analysis.

\subsection{Stability of symmetrical beams, loaded in compression and biaxial bending.}

\subsubsection{General differential equations}

From equilibrium of a deformed element, the general differential equations are given by Chen and Atsuta ([2], eq.(2.179a)). For symmetrically beams these simplify to (see notations):

$$
\begin{aligned}
& E I_{y} w^{\prime \prime \prime+}+F w^{\prime \prime}+\left(\varphi M_{z}\right) "-v^{\prime \prime} M_{t}-2 v^{\prime \prime} M_{t}{ }^{\prime}+M_{y} "=0 \ldots \\
& E I_{z} v^{\prime \prime \prime}+F v^{\prime \prime}+\left(\varphi M_{y}\right) "-w^{\prime \prime \prime} M_{t}-2 w^{\prime \prime} M_{t}{ }^{\prime}+M_{z} "=0 \ldots \\
& E I_{w} \varphi^{\prime \prime \prime}-\left(G I_{t}+K\right) \varphi "+v^{\prime \prime} M_{y}+w^{\prime \prime} M_{z}-v \cdot M_{y} "-w \cdot M_{z} "+M_{t}{ }^{\prime}=0 \ldots
\end{aligned}
$$

Further simplification is possible by omitting small terms. This can be seen as follows by using the first term of the Fourier expansion of the variables.

For simply supported beams is for instance: $v=\bar{v} \sin (\pi x / L)$ and $M_{t}=\bar{M}_{t} \cos (\pi x / L)$ and the term: $2 v^{\prime \prime} M_{t}{ }^{\prime}$ of eq.(46) has a maximum value of order: $\bar{v} \cdot \bar{M}_{t} \pi^{3} / L^{3}$. Also the maximum value of $v^{\prime \prime} M_{t}{ }^{\prime}$ is of this order. As shown later, the top-value of $2 v^{\prime \prime} M_{t}{ }^{\prime}$ is: $\left(\pi^{2} / L^{2}\right) \bar{v}(\bar{p} h+\bar{q} b)$, what is negligible with respect to: $M_{y} "=q$ in eq.(46), because $\bar{v}<<b$ and $b<L$. In the same way, it can be shown that, when $\mathrm{q}=0$, the term: $2 v^{\prime \prime} M_{t}{ }^{\prime}$ is small with respect to the terms $\left(\varphi M_{z}\right) "$ and $E I_{y} w^{\prime \prime \prime \prime}$ and the terms with $M_{t}$ and $M_{t}{ }^{\prime}$ can be omitted in eq.(46) and for the same reason also in eq.(47). The values in eq.(48) $w^{\prime \prime} M_{z}$ and $w \cdot M_{z}$ " are also comparable and equal to $\left(\pi^{2} / L^{2}\right) \bar{w} \cdot \bar{M}_{z} \sin (\pi x / L)$ and in the same way is $v^{\prime \prime} M_{y} \quad v M_{y} "-q v$.

From Fig. 8, (or Fig. 1 of [11]) follows that the increase of the torsional moment per unit length is:

$-M_{t}{ }^{\prime}=p s_{p}+q s_{q}-p w+q v+p \varphi e_{p}+q \varphi e_{q} \ldots$

Thus: $2 v^{\prime \prime} M_{t}^{\prime} \quad 2 v\left(\pi^{2} / L^{2}\right)\left(p s_{p}+q s_{q}-p w+q v+p \varphi e_{p}+q \varphi e_{q}\right)$, and for high eccentricities, e.g. $s_{p}=h / 2$ and $s_{q}=b / 2$, the terms $p h / 2+q b / 2$ dominate because $w<</ 2$; $v<<b / 2 ; \varphi e_{p}<b / 2$ giving:

$2 v M_{t}{ }^{\prime} \approx v\left(\pi^{2} / L^{2}\right)(p h+q b) \ldots$

For small eccentricities, e.g. for $s_{p}=s_{q}=0$, this term $2 v M_{t}{ }^{\prime}$ is much smaller and it follows that this term is always negligible. 
In eq.(48), $\varphi^{\prime \prime \prime \prime}$ can be replaced by $\varphi^{\prime \prime \prime}=-\left(\pi^{2} / L^{2}\right) \varphi$ ", in the usually applied order, giving:

$$
\left(E I_{w}\left(\pi^{2} / L^{2}\right)+G I_{t}+K\right) \varphi^{\prime \prime}-v^{\prime \prime} M_{y}-q \cdot v-M_{t}{ }^{\prime}=0 \ldots
$$

According to eq.(49) is:

$-M_{t}{ }^{\prime}-q v=p s_{p}+q s_{q}-p w+p \varphi e_{p}+q \varphi e_{q}=p s_{v}+q \varphi e_{v}-p w$ and with:

$s_{v}=s_{p}\left(1+q s_{q} / p s_{p}\right)$ and: $e_{v}=e_{q}\left(1+p e_{p} / q e_{q}\right)$, and with:

$G I_{v}=\frac{\pi^{2}}{L^{2}} E I_{w}+G I_{t}+K=G I_{t}\left(1+\frac{\pi^{2}}{L^{2}} \frac{E I_{w}}{G I_{t}}-\frac{F\left(I_{y}+I_{z}\right)}{G I_{t} A}\right)=G I_{t}\left(1+\frac{\pi^{2}}{L^{2}} \frac{E I_{w}}{G I_{t}}\right)\left(1-\frac{F}{F_{t}}\right)$,

where: $F_{t}=\frac{G I_{t} A}{I_{y}+I_{z}}\left(1+\frac{\pi^{2}}{L^{2}} \frac{E I_{w}}{G I_{t}}\right)$ is the twist buckling force, eq.(51) becomes:

$G I_{v} \varphi "-v^{\prime \prime} M_{y}+p s_{v}+q \varphi e_{v}-p w=0 \ldots$

For high beams the term: $p w$ is small and can be neglected in eq.(52).

For high beams, $I_{y}>>I_{z}$ and thus $p<<q$, is also the term: $\left(\varphi M_{z}\right) "$ negligible in eq.(46)

because: $\left(\varphi M_{y}\right)^{\prime \prime} \approx 4 p \varphi<M_{y} "=-q$

However in eq.(47) is, for high beams, $\left(\varphi M_{y}\right) " \approx 4 q \varphi$ not always of lower order than: $-p$

or $E I_{z} v^{\prime \prime \prime}$ and can only be neglected for low beams. For high beams, eq.(46) to eq.(48) are:

$E I_{y} w^{\prime \prime \prime}+F w^{\prime \prime}-q=0 \ldots$

$E I_{z} v^{\prime \prime \prime+}+F v^{\prime \prime}+\left(\varphi M_{y}\right) "-p=0 \ldots$

$G I_{t} \varphi^{\prime \prime}-v \cdot M_{y} "+q \cdot \varphi \cdot e_{v}=0 \ldots$

Eq.(46) is uncoupled and can be solved directly. This result is similar to bifurcation behavior and shows that the same loading path for failure is critical, and can be followed, as applies for elastic bifurcation. Thus, first allow only vertical movement $w$ in z-direction by choosing $\varphi$ and $v$ identical to zero, leading to eq.(46'). Then, at constant $w$, allow lateral buckling according to eqs. $(47,48)$ and solve eq. $\left(46^{\prime}\right)$ separately and use the result in eqs. $(47,48)$. This will be shown in Section 3.2.2.

For low beams, where $I_{y}$ and $I_{z}$ are not far apart, eqs. $(46,47,48)$ become:

$E I_{y} w^{\prime \prime \prime}+F w^{\prime \prime}-q=0 \ldots$

$E I_{z} v^{\prime \prime \prime}+F v^{\prime \prime}-p=0 \ldots$

$G I_{t} \varphi "-v \cdot M_{y} "+q \cdot \varphi \cdot e_{v}-p w=0 \ldots$

Now also the second equation is uncoupled and this result can be inserted in eq.(48' ') to show that also this equation is uncoupled when $I_{z} \rightarrow I_{y}$. Eq.(48) can be written:

$G I_{v} \varphi^{\prime \prime}+q v-p w+p s_{p}+q s_{q}+p e_{p} \varphi+q e_{q} \varphi=0$, and now is:

$q v-p w=q v\left(1-\frac{p v}{q u}\right)=q u \frac{1-E I_{Z} / E I_{Y}}{1-F / F_{e y}}$, because according to eq.(46"') and eq.(47')):

$\frac{p w}{q v}=\frac{\left(E I_{z} \pi^{4} / L^{4}-F \pi^{2} / L^{2}\right) v w}{\left(E I_{y} \pi^{4} / L^{4}-F \pi^{2} / L^{2}\right) v w}=\frac{E I_{z}}{E I_{y}} \frac{1-F / F_{e z}}{1-F / F_{e y}}=\frac{E I_{Z} / E I_{Y}-F / F_{e y}}{1-F / F_{e y}}$,

where $F_{e y}=\left(\pi^{2} / L^{2}\right) E I_{y}$ and $F_{e z}=\left(\pi^{2} / L^{2}\right) E I_{z}$.

Eq.(48') now can be written, with $q v=-M_{y} " v=-M_{y} v "$ : 
$\frac{G I_{v}\left(1-F / F_{e y}\right)}{1-E I_{z} / E I_{y}} \varphi^{\prime \prime}-M_{y} v^{\prime \prime}+\frac{\left(1-F / F_{e y}\right)}{1-E I_{z} / E I_{y}}\left(p s_{v}+q e_{v} \varphi\right)=0 \ldots$

or: $G I_{m} \varphi "-M_{y} v^{\prime \prime}+p s_{m}+q e_{m} \varphi=0 \ldots$

where $G I_{v}, s_{v}$, and $e_{v}$ are multiplied by $\left(1-F / F_{e y}\right) /\left(1-E I_{z} / E I_{y}\right)$ to get $G I_{m}, s_{m}, e_{m}$.

It follows from eq.(53) that for $I_{z} \rightarrow I_{y}$, the first and third terms dominate and thus $M_{y} v$ " vanishes and thus also the coupling ends and the equation for pure torsional twist remains:

$G I_{v} \varphi "+q e_{v} \varphi+p s_{v}=0 \ldots$

Thus equation (46'), (47') and (53') now apply in general for high as well as low beams.

Inserting the initial eccentricities $v_{0}, w_{0}, \varphi_{0}$ these equations become:

$E I_{y}\left(w^{\prime \prime \prime}-w_{0}{ }^{\prime \prime \prime}\right)+F w^{\prime \prime}-M_{y}{ }^{\prime \prime}=0 \ldots$

$E I_{z}\left(v^{\prime \prime \prime}-v_{0}{ }^{\prime \prime \prime}\right)+F v^{\prime \prime}+\left(\varphi M_{y}\right) "+M_{y}^{\prime}=0 \ldots$

$G I_{m}\left(\varphi^{\prime \prime}-\varphi_{0} "\right)+q e_{m} \varphi-M_{y} v^{\prime \prime}+p s_{m}=0 \ldots$

where eq. $\left(48^{\prime \prime \prime}\right)$ is at the safe side when $\varphi_{0}$ is important. The differential equations are now expressed in the usual applied form, but differ from these by the equivalent eccentricities and rigidity $G I_{m}$ by accounting for warping effects.

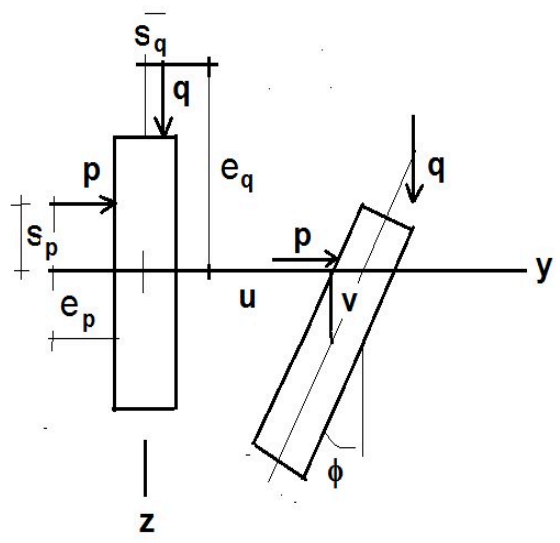

\section{2.2 Solution of the differential equations}

Because every loading case is different in practice, and superposition is not allowed, it would be necessary to repeat the solution for every case. This can be, and is avoided here, by a solution based on the first expanded of the Fourier expansion of the loading. Then for any load combination the solution is known differing from each other by the resulting top value of the expanded terms. Eq.(46) is directly solvable.

Fig. 8. Loading and displacements of a beam cross section

With $w=\bar{w} \sin (\pi x / L) ; w_{0}=\bar{w}_{0} \sin (\pi x / L)$, and $q=\bar{q} \sin (\pi x / L)$, is eq.(46','):

$\bar{w}=\frac{F_{e y} \bar{w}_{0}+\bar{M}_{y}}{F_{e y}-F} \quad$ with: $\bar{M}_{y}=\bar{q} L^{2} / \pi^{2}$ and: $F_{e y}=\pi^{2} E I_{y} / L^{2}$

Because $M_{y F}=-E I_{y}\left(w-w_{0}\right)^{\prime \prime}$, with top-value: $\bar{M}_{y F}=\left(\pi^{2} / L^{2}\right) E I_{y}\left(\bar{w}-\bar{w}_{0}\right)$, is:

$M_{y F}=\frac{F w_{0}+M_{y}}{1-F / F_{e y}} \ldots$

The solution of the coupled eq.(47',') and eq.(48,' $)$ is possible by the use of the virtual work equation, which also is the basis of theorems of limit analysis as the normality rule and the upper and lower bound method. The total work of an equilibrium system which is subjected to a possible virtual deformation remains zero. Thus for the differential equation (47'") and $\left(48^{\prime \prime \prime}\right): \bar{L}_{2}(v, \varphi)=0$ and $\bar{L}_{3}(v, \varphi)=0$ applies: $\int \bar{L}_{2}(v, \varphi) f_{i}(x) d x=0$ and $\int \bar{L}_{3}(v, \varphi) f_{i}(x) d x=0$, with $f_{i}=\sin (\pi x / L)$ as possible virtual displacement satisfying the boundary conditions. 
These equations thus are solved for the first expanded of the Fourier sinus series of deformation and loading:

$v=\bar{v} \sin (\pi x / L), v_{0}=\bar{v}_{0} \sin (\pi x / L), \varphi=\bar{\varphi} \sin (\pi x / L), \varphi_{0}=\bar{\varphi}_{0} \sin (\pi x / L), p=\bar{p} \sin (\pi x / L)$ $M_{z}=\bar{M}_{z} \sin (\pi x / L)$, where $p=M_{z} "=d^{2}\left(M_{z}\right) / d x^{2}$.

For the main loading also a second expanded term is accounted to show its small influence.

Thus: $M_{y}=M_{1} \sin (\pi x / L)+M_{3} \sin (3 \pi x / L)$ and: $q=q_{1} \sin (\pi x / L)+q_{3} \sin (3 \pi x / L)$

$\int_{0}^{L} \bar{L}_{2}(v, \varphi) f_{i}(x) d x=\int_{0}^{L}\left[E I_{z} \frac{\pi^{4}}{L^{4}}\left(\bar{v}-\bar{v}_{0}\right) \sin ^{2}\left(\frac{\pi x}{L}\right)-F \bar{v} \frac{\pi^{2}}{L^{2}} \sin ^{2}\left(\frac{\pi x}{L}\right)-\bar{\varphi} \frac{\pi^{2}}{L^{2}} \sin ^{2}\left(\frac{\pi x}{L}\right)\right.$.

$\cdot\left\{M_{1} \sin \left(\frac{\pi x}{L}\right)+M_{3} \sin \left(\frac{3 \pi x}{L}\right)\right\}+2 \frac{\pi^{2}}{L^{2}} \bar{\varphi} \cos \left(\frac{\pi x}{L}\right) \sin \left(\frac{\pi x}{L}\right) \cdot\left\{M_{1} \cos \left(\frac{\pi x}{L}\right)+3 M_{3} \cos \left(\frac{3 \pi x}{L}\right)\right\}$

$\left.+\bar{\varphi} \frac{\pi^{2}}{L^{2}} \sin ^{2}\left(\frac{\pi x}{L}\right) \cdot\left\{M_{1} \sin \left(\frac{\pi x}{L}\right)+9 M_{3} \sin \left(\frac{3 \pi x}{L}\right)\right\}-M_{z} \frac{\pi^{2}}{L^{2}} \sin ^{2}\left(\frac{\pi x}{L}\right)\right] \cdot d x=0$

$\int_{0}^{L} \bar{L}_{3}(v, \varphi) f_{i}(x) d x=\int_{0}^{L}\left[-G I_{m} \frac{\pi^{2}}{L^{2}}\left(\bar{\varphi}-\bar{\varphi}_{0}\right) \sin ^{2}\left(\frac{\pi x}{L}\right)+\bar{v} \frac{\pi^{2}}{L^{2}} \sin ^{2}\left(\frac{\pi x}{L}\right) \cdot\left\{M_{1} \sin \left(\frac{\pi x}{L}\right)+\right.\right.$

$\left.\left.+M_{3} \sin \left(\frac{3 \pi x}{L}\right)\right\}+e_{m} \bar{\varphi} \sin ^{2}\left(\frac{\pi x}{L}\right)\left\{q_{1} \sin \left(\frac{\pi x}{L}\right)+q_{3} \sin \left(\frac{3 \pi x}{L}\right)\right\}+\bar{p} s_{m} \sin ^{2}\left(\frac{\pi x}{L}\right)\right] d x=0$

In these equations are:

$\frac{\pi}{L} \int_{0}^{L} \sin ^{2}\left(\frac{\pi x}{L}\right) d x=\int_{0}^{L} \sin ^{2}\left(\frac{\pi x}{L}\right) d\left(\frac{\pi x}{L}\right)=\int_{0}^{\pi} \sin ^{2}(\alpha) d \alpha=\frac{\pi}{2}$

$\int_{0}^{\pi} \sin ^{3}(\alpha) d \alpha=\frac{4}{3} ; \int_{0}^{\pi} \sin ^{2}(\alpha) \sin (3 \alpha) d \alpha=-\frac{4}{15} ; \int_{0}^{\pi} \sin (\alpha) \cos ^{2}(\alpha) d \alpha=\frac{2}{3}$;

$\int_{0}^{\pi} \sin (\alpha) \cos (\alpha) \cos (3 \alpha) d \alpha=-\frac{2}{5}$. Thus the equations become:

$E I_{z} \frac{\pi^{4}}{L^{4}}\left(\bar{v}-\bar{v}_{0}\right) \frac{\pi}{2}-F \bar{v} \frac{\pi^{2}}{L^{2}} \frac{\pi}{2}-\bar{\varphi} \frac{\pi^{2}}{L^{2}} M_{1} \frac{4}{3}+\bar{\varphi} \frac{\pi^{2}}{L^{2}} M_{3} \frac{4}{15}+2 \frac{\pi^{2}}{L^{2}} \bar{\varphi} M_{1} \frac{2}{3}-2 \frac{\pi^{2}}{L^{2}} \bar{\varphi} 3 M_{3} \frac{2}{5}$

$-\bar{\varphi} \frac{\pi^{2}}{L^{2}} M_{1} \frac{4}{3}+9 M_{3} \bar{\varphi} \frac{\pi^{2}}{L^{2}} \frac{4}{15}-\bar{M}_{z} \frac{\pi^{2}}{L^{2}} \frac{\pi}{2}=0$, and:

$-G I_{m} \frac{\pi^{2}}{L^{2}}\left(\bar{\varphi}-\bar{\varphi}_{0}\right) \frac{\pi}{2}+\bar{v} \frac{\pi^{2}}{L^{2}} M_{1} \frac{4}{3}-\bar{v} \frac{\pi^{2}}{L^{2}} M_{3} \frac{4}{15}+e_{m} \bar{\varphi} q_{1} \frac{4}{3}-e_{m} \bar{\varphi} q_{3} \frac{4}{15}+\bar{p} S_{m} \frac{\pi}{2}=0$

Thus: $E I_{z} \frac{\pi^{2}}{L^{2}}\left(\bar{v}-\bar{v}_{0}\right)-F \bar{v}-\bar{\varphi} M_{1} \frac{8}{3 \pi}+\bar{\varphi} M_{3} \frac{8}{15 \pi}-\bar{M}_{z}=0$, and:

$-G I_{m}\left(\bar{\varphi}-\bar{\varphi}_{0}\right)+\bar{v} M_{1} \frac{8}{3 \pi}-\bar{v} M_{3} \frac{8}{15 \pi}+e_{m} \bar{\varphi} q_{1} \frac{L^{2}}{\pi^{2}} \frac{8}{3 \pi}-e_{m} \bar{\varphi} q_{3} \frac{L^{2}}{\pi^{2}} \frac{8}{15 \pi}+\bar{p} \frac{L^{2}}{\pi^{2}} S_{m}=0$.

With: $q_{e}=\frac{8}{3 \pi} \bar{q}_{1}\left(1-\frac{\bar{q}_{3}}{5 \bar{q}_{1}}\right) ; \quad M_{y, e}=\frac{8}{3 \pi} M_{1}\left(1-\frac{M_{3}}{5 M_{1}}\right) ; \quad F_{e z}=\frac{\pi^{2}}{L^{2}} E I_{z} ; \quad e_{m}^{1}=e_{m} \frac{q L^{2}}{\pi^{2} M_{y}}$

and: $s_{m}^{1}=s_{m} \frac{\bar{p} L^{2} / \pi^{2}}{\bar{M}_{z}}$, are these equations:

$F_{e y}\left(\bar{v}-\bar{v}_{0}\right)-F \bar{v}-\bar{\varphi} M_{y, e}-\bar{M}_{z}=0 \ldots$

$G I_{m}\left(\bar{\varphi}-\bar{\varphi}_{0}\right)-M_{y, e} \bar{v}-e_{m}^{1} \bar{\varphi} M_{y, e}-s_{m}^{1} \bar{M}_{z}=0 \ldots$

From these last two equations, $\bar{v}$ and $\bar{\varphi}$ can be resolved, giving: 


$$
v=\frac{\bar{M}_{z}\left(G I_{m}-e_{m}^{1} M_{y, e}+s_{m}^{1} M_{y, e}\right)+M_{y, e} G I_{m} \bar{\varphi}_{0}+F_{e y} \bar{u}_{0}\left(G I_{m}-e_{m}^{1} M_{y, e}\right)}{\left(F_{e y}-F\right)\left(G I_{m}-e_{m}^{1} M_{y, e}\right)-M_{y, e}^{2}} \ldots
$$

and because $M_{z, F}=-E I_{z}\left(v-v_{0}\right) "=\frac{\pi^{2}}{L^{2}} E I_{z}\left(\bar{v}-\bar{v}_{0}\right)=F_{e z}\left(\bar{v}-\bar{v}_{0}\right)$, is:

$$
M_{z, F}=\frac{\bar{M}_{z}\left(G I_{m}+\left(s_{m}^{1}-e_{m}^{1}\right) M_{y, e}\right)+M_{y, e} G I_{m} \varphi_{0}+M_{y, e}^{2} v_{0}+F_{e y} v_{0}\left(G I_{m}-e_{m}^{1} M_{y, e}\right)}{\left(F_{e z}-F\right)\left(G I_{m}-e_{m}^{1} M_{y, e}\right)-M_{y, e}^{2}} F_{e z} \ldots
$$

with $\bar{M}_{z}$ and $\bar{M}_{y, e}$ of the first order moments.

In Table 1, values of $M_{y, e}$ are given based on the first expanded: $8 \bar{M} / 3 \pi$ and based on the full series (or exact), and based on the mean value of $M$ of middle half of the beam (the part that deflects the most at buckling). Because the virtual work equation follows $\sin ^{2}(\pi x / L)$ along the length of the beam, giving the value one at the middle half of the beam and the value of about zero over the other parts, only the middle part accounts for the work done.

\section{Conclusions}

- The given equations of the biaxial bending strength are in accordance with the limit analysis method and thus based on elastic-full-plastic behavior. Therefore, with the restriction of applying a mean hardening stress after initial "flow", the analysis is rigorous and the strength prediction realistic and the result has to be applied in the Building Codes to provide the by Euro-law prescribed sufficient precise reliability calculation (also for totally new, never occurred and never measured, cases).

- For the highest lower bound solution of biaxial bending strength is necessary that the neutral axis is a straight line and that unlimited flow in pure compression occurs, thus when there is bending-tension failure and when the shear stress is carried in the elastic part of the cross section. This is an improvement with respect to the thus far applied, (not unique) old model of [7] restricting the ultimate plastic compression strain at failure.

- The derived general expressions in coordinates of the boundary line of the full compression area provide 3 cases for design. For simplicity of design, is chosen for separate ultimate shear strength and ultimate bending-compression strength equations.

- The equations contain also the solution for uniaxial bending cases, which are already shown to precisely explain and fit data by the applied elastic full plastic limit analysis.

- The value of $s=f_{t} / f_{c}$ appears to be about constant for all determining load combinations of bending with compression, indicating again (by the data of [7]) that there always is failure by the ultimate tensile strength. A volume effect by stress distribution thus needs not to be regarded as follows from the uniaxial data. The volume effect thus now is caused by the volume alone due to decreasing quality by volume increase.

- The solutions of the most general equilibrium equations, eq.(54) and eq.(58) are exact, complete and universal, applicable for any material and load combination, based on the virtual work principle, which also is the basis of the lower and upper bound solutions of limit analysis and which always provides an exact solution however complex the equilibrium equations are. The equilibrium equations have to satisfy the mentioned biaxial failure criterion of the stability problem, which is always a strength problem for full scale timber beams as empirically verified in the past. 
Table B1. Values of $M_{y e}$

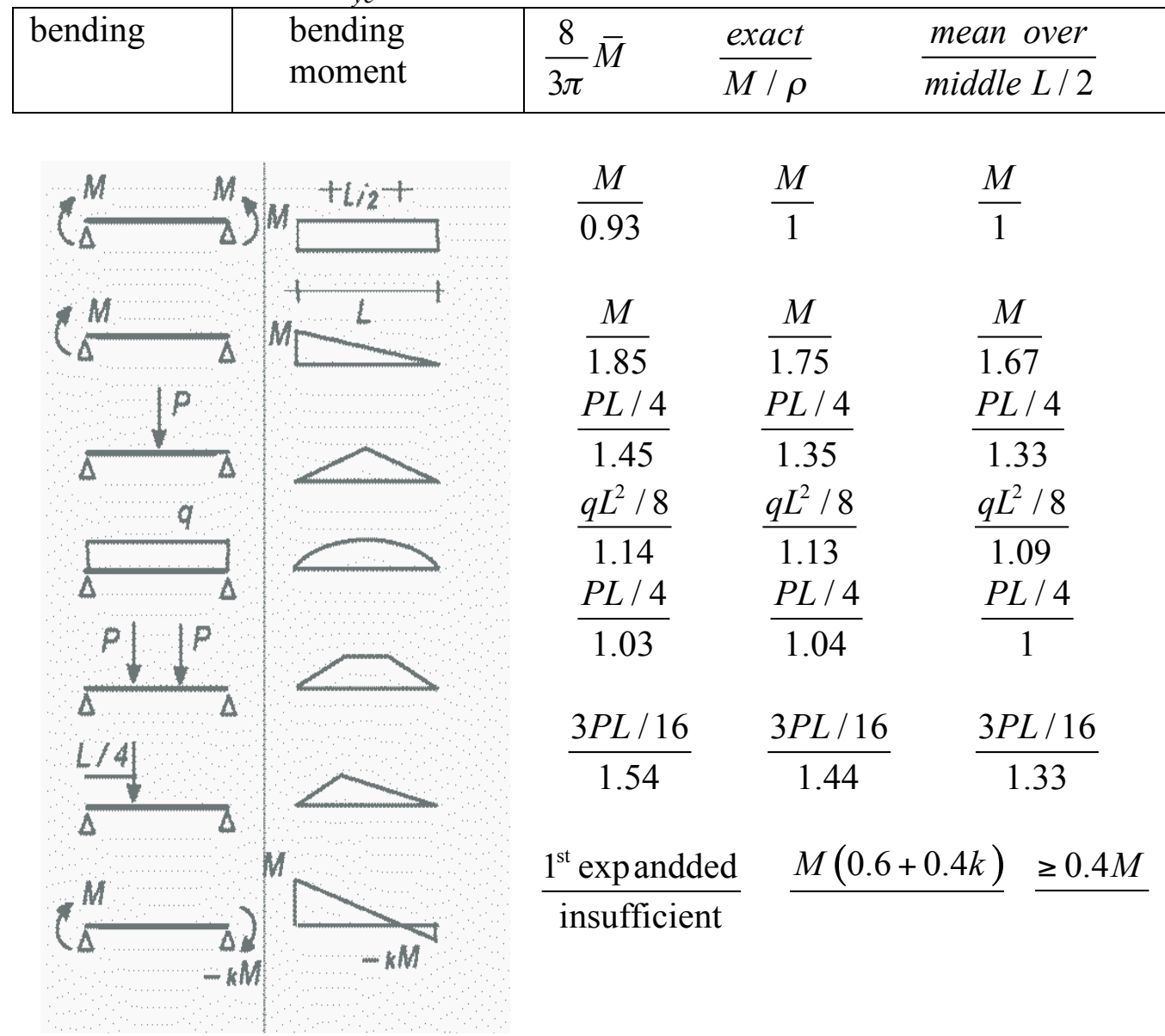

\section{Notations}

$A \quad$ area of the cross section of the beam

$b, h \quad$ beam dimensions

$E I_{y}, E I_{z}$ bending rigidity about the y-axis and z-axis

$E I_{w} \quad$ warping rigidity

$e, s \quad$ eccentricity of the lateral loading

$F \quad$ normal force

$F_{t} \quad$ twist buckling force: $F_{t}=G I_{t} A\left(1+\pi^{2} E I_{w} /\left(L^{2} G I_{t}\right)\right) /\left(I_{y}+I_{z}\right)$

$f_{t}, f_{c}, f_{v}$ real ultimate tensile, compression and shear stress

$f_{m}, f_{v, f} \quad$ ultimate fictive, linearized, bending and shear stress of the Codes.

$f_{m}=f_{c}(3 s-1) /(s+1) ; \quad f_{v, f}=2 f_{v} /(s+1)$

$G I_{t} \quad$ torsional rigidity ( St. Venant)

$G I_{v} \quad$ equivalent torsional rigidity for high beams $=G I_{t}\left(1+\pi^{2} E I_{w} /\left(L^{2} G I_{t}\right)\right)\left(1-F / F_{t}\right)$

$G I_{m} \quad$ equivalent torsional rigidity $=G I_{v}\left(1-F / F_{e y}\right) /\left(1-E I_{z} / E I_{y}\right)$

$K \quad$ Wagner effect $=-F\left(I_{y}+I_{z}\right) / A$

$L \quad$ span, or effective buckling length

$M_{y}, M_{z}$ bending moments about resp. the y-axis and z-axis

$M_{y}{ }^{\prime} \quad$ derivative of $M_{y}$ to $x$, 


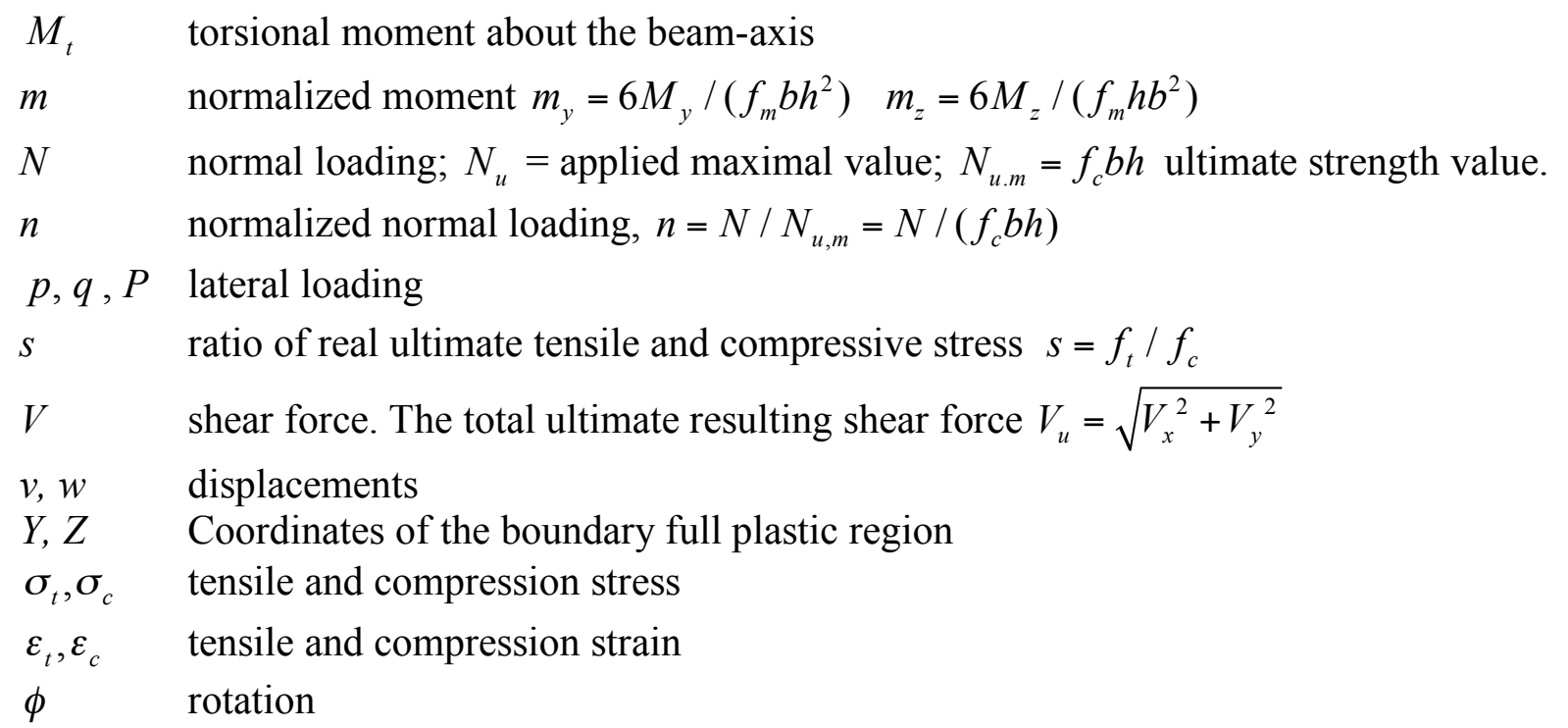

\section{References}

[1] T.A.C.M. van der Put, "Derivation of the bi-axial bending, compression and shear strengths of timber beams", The open mechanics Journal, pp 14-21, 2012.

[2] W.-F. Chen, T. Atsuta, Theory of beam-Columns, Vol. 2: Space behavior and Design, J. Ross Publishing, 2007.

[3] T.A.C.M. van der Put, "Failure criterion for timber beams loaded in bending, compression and shear", Wood Material Science and Engineering,; 1: pp 41-49, 2010.

[4] Eurcode 5, EN 1995: 2004 Design of Timber Structures, Part 1-1: General - Common rules and rules for buildings

[5] F. Kollmann, Technologie des Holzes und der Holzwerkstoffe Springer Verlag Berlin 1951.

[6] T.A.C.M. van der Put, "Derivation of the Bi-axial Bending, Compression and Shear Strengths of Timber Beams", The Open Mechanics Journal, 6, pp 14-21 2012,

[7] K.C. Johns, A.H. Buchanan "Strength of timber members in combined bending and axial loading”, CIB/IUFRO Meeting Boras, Sweden, 1982. (see also CIB W18, 1985 Israel).

[8] NEN 6760 - TGB 1990 - Timber structures - Basic requirements and determination methods, NNI 1990.

[9] T.A.C.M. van der Put, J.W.G. van de Kuilen, "Derivation of the shear strength of continuous beams", European Journal of Wood and Wood Products 69, pp 421-430, 2010

[10] T.A.C.M. van der Put, "A continuum failure criterion applicable to wood", J. Wood Sci 55: 315-322, 2009.

[11] T.A.C.M. van der Put. "Stability design and Code rules for straight timber beams", Proc. CIB - W18/23-15-2, Portugal, 1990

which is based on the Technical Report 4-81-11 of the Stevin-laboratory, 1981, and is applied and approved during decades as the Dutch Building regulations, TGB. 